\title{
Bilateral Teleoperation Using Unknown Input Observers for Force Estimation
}

\author{
John M. Daly and David W. L. Wang
}

\begin{abstract}
Teleoperation systems that are distributed over large physical distances where time delays become nonnegligible are becoming popular in areas such as remote medicine and operating in hazardous environments. This work proposes a new approach to the bilateral control problem that extends an earlier approach [1] based on a sliding mode controller. The existing approach requires full state and force measurements at both the master and slave sides. Here, we propose the use of unknown input sliding mode observers to estimate the states and forces and use the estimates in the controllers. This significantly reduces the difficulty of implementation and the cost of such a system, as one need only measure positions in the system. The velocity and force signals are then estimated. A proof of stability for this new approach is given. As well, the approach is verified through a numerical simulation study as a precursor to ongoing experimental verification.
\end{abstract}

Index Terms-Sliding mode control, sliding mode observer, bilateral teleoperation, unknown input estimation

\section{INTRODUCTION}

Teleoperation is an area that holds a significant amount of interest to researchers. There can be great benefits to being able to operate machines from a distance. One example is the ability to send a robotic vehicle which is remotely controlled into a hazardous environment. Another useful application of teleoperation is in the field of remote medicine. A highly skilled surgeon could perform surgery on a patient who is located in another city, or even country. A doctor could also interact with a patient located somewhere else through a robotic interface in order to make a diagnosis. Another example of a teleoperated system is in Earth-tospace operations, where someone on the surface of the Earth controls a robot in space [2]. Note that not all of these applications consider force feedback, though systems using force feedback are of importance in this work.

When designing such teleoperation systems, one must consider which signals to pass between the master manipulator and the slave manipulator. The work here considers bilateral teleoperation, which involves some force feedback from the slave side to the master side. Two common bilateral teleoperation approaches are the position-position and the positionforce architectures [3]. In the former approach, the master position is passed to the slave side, and the slave position is passed back to the master side. Position controllers at both the master and slave sides ensure that the two manipulators follow each other. This approach is not particularly desirable in cases of free motion, since differences between the master

J. Daly and D. Wang are with the Department of Electrical and Computer Engineering, University of Waterloo, 200 University Avenue West, Waterloo, Ontario, Canada. jmdalydieee.org and slave position can cause the operator at the master side to feel large reaction forces [3], which are not due to any environmental contact at the slave side. However, no force sensors are required for such a system. One well known implementation of a variant of the position-position architecture is the wave variables approach of Niemeyer and Slotine [4].

In contrast, the position-force architecture requires force sensing at the slave side. However, the advantages to this are perfect force tracking and a better perception of the system in free motion than the position-position architecture provides [5]. The increased performance, from a transparency point of view, of the position-force architecture motivates research into teleoperation systems where the forces are measured. However, force sensors are costly, can be unreliable, and provide noisy signals. Therefore, this work seeks to develop an approach where the benefits of a teleoperation architecture using force sensors are obtained, without the need to actually measure the forces.

The area of research involving unknown input observers provides a useful framework for the problem of estimating external forces acting on a robot manipulator. Unknown input observers are commonly used when estimating the state of systems subject to disturbances and in the area of fault detection and diagnosis [6]. In the context of robotics and teleoperation, one may consider that a robot has two inputs - the control input and the force input applied to the robot by an external environment. The control input is clearly a known input, but without a force sensor the external force input may be viewed as an unknown input to be estimated. To the best of the authors knowledge, implementing unknown input observers as external force estimators has yet to be studied in the robotics field. The work presented here will study the estimation of the external forces on the master and slave robots in combination with a modified version of the sliding mode bilateral teleoperation algorithm, developed by Cho et al. [7], that normally requires both full state estimates and force sensors at both the master and slave sides. This work extends existing results in bilateral teleoperation by modifying an existing approach, shown to work well through experiments [7], such that only the position states need to be measured and force sensing is no longer required for the implementation. Additionally, a proof of stability of the new modified approach is presented.

In Section II, the concept of sliding mode unknown input observers is introduced. Section III presents the bilateral control algorithm of Cho et al. and the modification to the algorithm that makes use of the unknown input observers to 
estimate the plant states as well as human and environmental forces at the master and slave manipulators. A proof of stability is then presented. Simulation results showing the performance of this modified algorithm are given in Section IV. Finally, conclusions are presented in Section V.

\section{UNKNOWN INPUT SLIDING MODE OBSERVERS}

The field of unknown input observers is one that has received noteworthy attention from researchers over the years. These observers are useful in a number of situations, such as those in which estimation is being performed for a plant that is subject to unknown disturbances. In a classical observer these disturbances would affect the estimate of the state, but unknown input observers provide a framework to generate accurate state estimates despite the presence of disturbances.

Initial work on unknown input observers resulted in designs that require that a matching condition be satisfied. In particular, consider a linear time-invariant system,

$$
\begin{aligned}
\dot{x} & =A x+B u+D w \\
y & =C x
\end{aligned}
$$

where $u$ represents the known input vector and $w$ represents the unknown input vector. In designing a "standard" unknown input observer, the system must satisfy the condition $\operatorname{rank}(C D)=\operatorname{rank}(D)$. This condition effectively requires that the state that is directly coupled to the unknown inputs must be available from the measurements [8]. In the case of the robot systems considered here, this is not the situation. The external force input, considered the unknown input in this case, comes in through the velocity state, but it is typical to measure position only. However, a class of unknown input sliding mode observers has been developed which do not depend on satisfying this rank condition [9]. Rather, the system being observed must be in the so-called block triangular observer form. Robot dynamics are easily expressed in this form. Consider a second order mass-springdamper plant of the form,

$$
\begin{aligned}
\dot{x}_{1} & =x_{2} \\
\dot{x}_{2} & =-\frac{k}{m} x_{1}-\frac{b}{m} x_{2}+\frac{1}{m} u+\frac{1}{m} F \\
y & =x_{1}
\end{aligned}
$$

where $m, b$, and $k$ are the mass, damping constant, and spring constant of the system, $u$ represents a known input, and $F$ represents an unknown input. A step by step sliding mode observer for this plant may be designed as [10],

$$
\begin{aligned}
& \dot{\hat{x}}_{1}=\hat{x}_{2}+\lambda_{1} \operatorname{sgn}\left(y-\hat{x}_{1}\right) \\
& \dot{\hat{x}}_{2}=-\frac{k}{m} \hat{x}_{1}-\frac{b}{m} \hat{x}_{2}+\frac{1}{m} u+E_{1} \lambda_{2} \operatorname{sgn}\left(\tilde{x}_{2}-\hat{x}_{2}\right)
\end{aligned}
$$

where $\hat{x}_{1}$ and $\hat{x}_{2}$ are the estimate position and velocity, respectively, and $\tilde{x}_{2}=\hat{x}_{2}+\left(\lambda_{2} \operatorname{sgn}\left(y-\hat{x}_{1}\right)\right)_{e q}$. The term $E_{1}=0$ if $y-\hat{x}_{1} \neq 0$ and $E_{1}=1$ otherwise. The notation $(\cdot)_{e q}$ is used to denote a low pass filtering operation on the discontinuous switching term to obtain the equivalent output injection [11]. The equivalent output injection is analogous to the notion of equivalent control in sliding mode control

It will be shown in Section III that for large enough choices of the sliding gains $\lambda_{1}$ and $\lambda_{2}$ the observer estimates will converge to the true states in finite time and the unknown input may be obtained from the observer as well.

Having presented the unknown input sliding mode observer that will be used in the teleoperation system, the next section will present the bilateral control algorithm of Cho et al. [1] and show the modification to make use of the unknown input observer.

\section{Sliding Mode Bilateral Teleoperation}

Sliding mode control is an appealing approach for controller design, due in part to its robustness to a certain class of uncertainties and disturbances [12]. Park and Cho [7] and Cho et al.[1] make use of sliding mode control in the context of bilateral time-delayed teleoperation in order to ensure stable teleoperation despite the presence of the time delays. This bilateral control algorithm makes use of an impedance controller at the master side and a sliding mode controller at the slave side. However, the approaches presented in [7], [1] assume that the full state of both robots is measured and that external forces are measured on both sides as well. By introducing unknown input sliding mode observers, as presented in Section II, the full state of both the master and slave as well as the external forces acting on each manipulator can be estimated from just position measurements.

This algorithm is presented in the context of a system with fixed time delays. For simplicity in development of the algorithm and proof, assume a constant delay of $T$ seconds that is equal in both directions. Consider the following dynamics for the master manipulator,

$$
\begin{aligned}
& \dot{x}_{m_{1}}=x_{m_{2}} \\
& \dot{x}_{m_{2}}=-\frac{k_{m}}{m_{m}} x_{m_{1}}-\frac{b_{m}}{m_{m}} x_{m_{2}}+\frac{1}{m_{m}} u_{m}+\frac{1}{m_{m}} F_{h}(9)
\end{aligned}
$$

where $m_{m}$ is the mass, $b_{m}$ is the damping constant, $k_{m}$ is the spring constant, $x_{m_{1}}$ is the master position, $x_{m_{2}}$ is the master velocity, $u_{m}$ is the master control input, and $F_{h}$ is the force applied by the human to the plant. The slave dynamics are expressed as,

$$
\begin{aligned}
& \dot{x}_{s_{1}}=x_{s_{2}} \\
& \dot{x}_{s_{2}}=-\frac{k_{s}}{m_{s}} x_{s_{1}}-\frac{b_{s}}{m_{s}} x_{s_{2}}+\frac{1}{m_{s}} u_{s}-\frac{1}{m_{s}} F_{e}
\end{aligned}
$$

where $m_{s}$ is the mass, $b_{s}$ is the damping constant, $k_{s}$ is the spring constant, $x_{s_{1}}$ is the slave position, $x_{s_{2}}$ is the slave velocity, $u_{s}$ is the slave control input, and $F_{e}$ is the force exerted on the slave by the environment.

In this teleoperation approach, the goals involve ensuring that the slave manipulator position tracks the delayed master position and remains stable despite the presence of time 
delay. This is accomplished using the sliding mode controller. As well, the environmental force acting on the slave is reflected back to the master so that the operator may experience the forces acting on the slave. An impedance controller at the master side is used to give the master manipulator a desired characteristic impedance.

In this system, the master sends its position, velocity, force exerted on it by the human, and a delayed copy of the slave force back to the slave side. The following notation for a signal $x(t)$ delayed by $T$ seconds is used,

$$
x^{d}=x(t-T)
$$

A signal delayed by twice the time delay is denoted,

$$
x^{d d}=x(t-2 T)
$$

The slave sends only the force exerted on it by the environment to the master. The master receives $F_{e}^{d}=F_{e}(t-T)$.

Defining the master-slave position and velocity tracking error as $e_{r_{1}}=x_{s_{1}}-x_{m_{1}}^{d}$ and $e_{r_{2}}=x_{s_{2}}-x_{m_{2}}^{d}$ respectively, a desired closed loop impedance model for the slave side is [1],

$$
I=\bar{m}_{s} \dot{e}_{r_{2}}+\bar{b}_{s} e_{r_{2}}+\bar{k}_{s} e_{r_{1}}-F_{e}=0
$$

When (12) is satisfied, the slave side has the desired closed loop impedance. In order to ensure that this desired impedance characteristic is satisfied, a sliding surface for the slave controller is defined as [1],

$$
s=\frac{1}{\bar{m}_{s}} \int_{0}^{t} I(\tau) d \tau=0
$$

Once the sliding mode controller has driven the system trajectories to $s=0$ then (12) will be satisfied [1] and the slave manipulator will have the desired closed loop behaviour.

However, this work examines output feedback control. In order to estimate the master states and human input force, we propose the following step by step sliding mode observer for the master side,

$$
\begin{aligned}
\dot{\hat{x}}_{m_{1}}= & \hat{x}_{m_{2}}+\lambda_{m_{1}} \operatorname{sgn}\left(x_{m_{1}}-\hat{x}_{m_{1}}\right) \\
\dot{\hat{x}}_{m_{2}}= & -\frac{k_{m}}{m_{m}} \hat{x}_{m_{1}}-\frac{b_{m}}{m_{m}} \hat{x}_{m_{2}}+\frac{1}{m_{m}} u_{m} \\
& +\lambda_{m_{2}} \operatorname{sgn}\left(\tilde{x}_{m_{2}}-\hat{x}_{m_{2}}\right)
\end{aligned}
$$

where $\tilde{x}_{m_{2}}=\hat{x}_{m_{2}}+\lambda_{m_{2}} \operatorname{sgn}\left(x_{m_{1}}-\hat{x}_{m_{1}}\right)_{e q}$. The terms $\lambda_{m_{1}}$ and $\lambda_{m_{2}}$ are the sliding mode gains. The slave observer dynamics are given as,

$$
\begin{aligned}
\dot{\hat{x}}_{s_{1}}= & \hat{x}_{s_{2}}+\lambda_{s_{1}} \operatorname{sgn}\left(x_{s_{1}}-\hat{x}_{s_{1}}\right) \\
\dot{\hat{x}}_{s_{2}}= & -\frac{k_{s}}{m_{s}} \hat{x}_{s_{1}}-\frac{b_{s}}{m_{s}} \hat{x}_{s_{2}}+\frac{1}{m_{s}} u_{s} \\
& +\lambda_{s_{2}} \operatorname{sgn}\left(\tilde{x}_{s_{2}}-\hat{x}_{s_{2}}\right)
\end{aligned}
$$

where $\tilde{x}_{s_{2}}=\hat{x}_{s_{2}}+\lambda_{s_{2}} \operatorname{sgn}\left(x_{s_{1}}-\hat{x}_{s_{1}}\right)_{e q}$. The terms $\lambda_{s_{1}}$ and $\lambda_{s_{2}}$ are the sliding mode gains.
The master side impedance controller is expressed as,

$$
\begin{aligned}
u_{m}= & \left(k_{m}-\frac{m_{m}}{\bar{m}_{m}} \bar{k}_{m}\right) \hat{x}_{m_{1}}+\left(b_{m}-\frac{m_{m}}{\bar{m}_{m}} \bar{b}_{m}\right) \hat{x}_{m_{2}} \\
& +\left(\frac{m_{m}}{\bar{m}_{m}}-1\right) \hat{F}_{h}-\frac{m_{m}}{\bar{m}_{m}} \hat{F}_{e}^{d}
\end{aligned}
$$

where $\bar{k}_{m}, \bar{b}_{m}$, and $\bar{m}_{m}$ represent the desired spring constant, damping constant, and mass parameters. This control law is a modification of the master control law presented in [1] that makes use of the estimated plant states and forces. The estimated forces $\hat{F}_{h}$ and $\hat{F}_{e}$ are obtained from the sliding mode observers as follows: $\hat{F}_{h}=m_{m}\left(\lambda_{m_{2}} \operatorname{sgn}\left(\tilde{x}_{m_{2}}-\hat{x}_{m_{2}}\right)\right)_{e q}$ and $\hat{F}_{e}=-m_{s}\left(\lambda_{s_{2}} \operatorname{sgn}\left(\tilde{x}_{s_{2}}-\hat{x}_{s_{2}}\right)\right)_{e q}$.

For the slave controller, an output feedback version of the impedance model is defined as,

$$
\hat{I}=\bar{m}_{s} \dot{\hat{e}}_{r_{2}}+\bar{b}_{s} \hat{e}_{r_{2}}+\bar{k}_{s} \hat{e}_{r_{1}}-\hat{F}_{e}=0
$$

where $\hat{e}_{r_{1}}=\hat{x}_{s_{1}}-\hat{x}_{m_{1}}^{d}$ and $\hat{e}_{r_{2}}=\hat{x}_{s_{2}}-\hat{x}_{m_{2}}^{d}$. Now, the sliding surface in the output feedback case is defined as,

$$
\begin{aligned}
\hat{s} & =\frac{1}{\bar{m}_{s}} \int_{0}^{t} \hat{I}(\tau) d \tau \\
& =\hat{e}_{r_{2}}+\int_{0}^{t}\left(\frac{\bar{b}_{s}}{\bar{m}_{s}} \hat{e}_{r_{2}}+\frac{\bar{k}_{s}}{\bar{m}_{s}} \hat{e}_{r_{1}}-\frac{1}{\bar{m}_{s}} \hat{F}_{e}\right) d \tau \\
& =0
\end{aligned}
$$

Then, the slave side sliding mode controller is given as,

$$
\begin{aligned}
u_{s}= & -m_{s}\left[\left(\frac{\bar{k}_{s}}{\bar{m}_{s}}-\frac{k_{s}}{m_{s}}\right) \hat{x}_{s_{1}}+\left(\frac{\bar{b}_{s}}{\bar{m}_{s}}-\frac{b_{s}}{m_{s}}\right) \hat{x}_{s_{2}}\right. \\
& +\left(\frac{\bar{k}_{m}}{\bar{m}_{m}}-\frac{\bar{k}_{s}}{\bar{m}_{s}}\right) \hat{x}_{m_{1}}^{d}+\left(\frac{\bar{b}_{m}}{\bar{m}_{m}}-\frac{\bar{b}_{s}}{\bar{m}_{s}}\right) \hat{x}_{m_{2}}^{d} \\
& -\left(\frac{1}{\bar{m}_{m}}-\frac{1}{m_{m}}\right) \hat{F}_{h}^{d}+\frac{1}{\bar{m}_{m}} \hat{F}_{e}^{d d}-\frac{1}{\bar{m}_{s}} \hat{F}_{e} \\
& +\left(\lambda_{s_{2}} \operatorname{sgn}\left(\tilde{x}_{s_{2}}-\hat{x}_{s_{2}}\right)\right)_{e q} \\
& \left.-\left(\lambda_{m_{2}} \operatorname{sgn}\left(\tilde{x}_{m_{2}}^{d}-\hat{x}_{m_{2}}^{d}\right)\right)_{e q}+K_{g} \operatorname{sat}(\hat{s} / \epsilon)\right]
\end{aligned}
$$

Note that in the slave control law a $\operatorname{sat}(\cdot)$ function is used for the sliding surface $\hat{s}$ instead of a $\operatorname{sgn}(\cdot)$ function. This is to eliminate the problem of chattering that is common in sliding mode control [12]. The parameter $\epsilon$ is the width of the boundary layer and determines the upper bound on $\hat{s}$ once it has reached the set $\{\hat{s} \leq \epsilon\}$. The term $K_{g}$ is the sliding mode gain which, when set large enough, drives the system trajectories to the boundary layer $\{\hat{s} \leq \epsilon\}$ and keeps them there for all time.

To summarize, for master plant (8), (9) and slave plant (10), (11) connected bilaterally through a time delay of $T$ seconds in each direction, the system may be controlled using the master control law (18) with master side observer (14), (15) and slave sliding mode control law (21) with slave side observer (16), (17).

In order to show stability of this system, the following assumption is made. 
Assumption 3.1: The external forces acting on both master and slave are bounded for all time with some known upper bounds.

The next theorem presents the main theoretical result of the paper, showing asymptotic stability of both the master and slave under output feedback with force estimation, and in the presence of time delays in the communications.

Theorem 3.1: Consider master plant (8), (9) and slave plant (10), (11) connected bilaterally through a time delay of $T$ seconds in each direction, with master control law (18), master side observer (14), (15) and slave sliding mode control law (21) with slave side observer (16), (17). Then, there exists a sliding mode controller gain

$$
\begin{aligned}
K_{g}> & \mid \lambda_{s_{2}} \operatorname{sgn}\left(\tilde{x}_{s_{2}}-\hat{x}_{s_{2}}\right)-\left(\lambda_{s_{2}} \operatorname{sgn}\left(\tilde{x}_{s_{2}}-\hat{x}_{s_{2}}\right)\right)_{e q} \\
& -\left(\lambda_{m_{2}} \operatorname{sgn}\left(\tilde{x}_{m_{2}}-\hat{x}_{m_{2}}\right)\right. \\
& \left.+\left(\lambda_{m_{2}} \operatorname{sgn}\left(\tilde{x}_{m_{2}}-\hat{x}_{m_{2}}\right)\right)_{e q}\right) \mid+\varepsilon_{g}
\end{aligned}
$$

where $\varepsilon_{g}>0$ and observer gains $\lambda_{s_{1}}, \lambda_{s_{2}}, \lambda_{m_{1}}$, and $\lambda_{m_{2}}$ such that the state estimates recover the true state in finite time, the master plant has the desired impedance model, and the slave plant dynamics converge to a boundary layer of width $\epsilon$ around the desired slave impedance model in finite time. The value of $\epsilon$ can be made arbitrarily small, and in the limit as $\epsilon$ approaches zero, the slave dynamics approach the desired slave impedance in finite time.

Proof: The first step is to show that the estimated states converge to the true states in finite time. The analysis is only shown for the slave side observer, but a similar result is obtained when analyzing the stability of the master side observer. Define the slave observer error as $e_{s_{1}}=x_{s_{1}}-\hat{x}_{s_{1}}$ and $e_{s_{2}}=x_{s_{2}}-\hat{x}_{s_{2}}$. Then, the slave observer error dynamics are expressed as,

$$
\begin{aligned}
\dot{e}_{s_{1}}= & e_{s_{2}}-\lambda_{s_{1}} \operatorname{sgn}\left(x_{s_{1}}-\hat{x}_{s_{1}}\right) \\
\dot{e}_{s_{2}}= & -\frac{k_{s}}{m_{s}} e_{s_{1}}-\frac{b_{s}}{m_{s}} e_{s_{2}}-\frac{1}{m_{s}} F_{e} \\
& -E_{1} \lambda_{s_{2}} \operatorname{sgn}\left(\tilde{x}_{s_{2}}-\hat{x}_{s_{2}}\right)
\end{aligned}
$$

Begin by showing stability of the first observer error state. Choose as a Lyapunov function candidate,

$$
V_{1}=\frac{1}{2} e_{s_{1}}^{2}
$$

Taking the derivative of the Lyapunov function along the trajectories of the system one arrives at,

$$
\begin{aligned}
\dot{V}_{1} & =e_{s_{1}} \dot{e}_{s_{1}}=-\lambda_{s_{1}}\left|e_{s_{1}}\right|+e_{s_{1}} e_{s_{2}} \\
& \leq-\lambda_{s_{1}}\left|e_{s_{1}}\right|+\left|e_{s_{1}}\right|\left|e_{s_{2}}\right| \\
& =-\left|e_{s_{1}}\right|\left(\lambda_{s_{1}}-\left|e_{s_{2}}\right|\right)
\end{aligned}
$$

Choosing $\lambda_{s_{1}}>\left|e_{s_{2}}\right|+\varepsilon_{1}$, where $\varepsilon_{1}>0$ yields $\dot{V}_{1} \leq$ $-\left|e_{s_{1}}\right| \varepsilon_{1}$. This ensures that $\dot{V}_{1}<0$ for all $e_{s_{1}} \neq 0$. Using the Comparison Lemma [12, p. 102] it may be shown that the surface $e_{s_{1}}=0$ is reached in finite time. An upper bound $T_{s_{1}}$ on this convergence time is determined from the Comparison Lemma as,

$$
T_{s_{1}} \triangleq \frac{\left|e_{s_{1}}\left(t_{0}\right)\right|}{\varepsilon_{1}}+t_{0}
$$

Therefore by the time $t=T_{s_{1}}$ the state $e_{s_{1}}$ will have converged to the sliding surface $e_{s_{1}}=0$. On that surface $e_{s_{1}}=\dot{e}_{s_{1}}=0$ and solving for the equivalent output injection yields $\left(\lambda_{s_{1}} \operatorname{sgn}\left(e_{s_{1}}\right)\right)_{e q}=e_{s_{2}}$. This results in $\tilde{x}_{s_{2}}=x_{s_{2}}$ on the sliding surface. Following [13], the equivalent control is obtained by a low pass filtering operation of $\lambda_{s_{1}} \operatorname{sgn}\left(e_{s_{1}}\right)$.

After time $T_{s_{1}}$ the slave observer error dynamics have the form,

$$
\begin{aligned}
\dot{e}_{s_{1}}= & e_{s_{2}}-\lambda_{s_{1}} \operatorname{sgn}\left(e_{1}\right)=0 \\
\dot{e}_{s_{2}}= & -\frac{k_{s}}{m_{s}} e_{s_{1}}-\frac{b_{s}}{m_{s}} e_{s_{2}}-\frac{1}{m_{s}} F_{e} \\
& -\lambda_{s_{2}} \operatorname{sgn}\left(e_{s_{2}}\right)
\end{aligned}
$$

We may then choose the Lyapunov function candidate $V_{2}=(1 / 2) e_{s_{1}}^{2}+(1 / 2) e_{s_{2}}^{2}=(1 / 2) e_{s_{2}}^{2}$, since at this point $e_{s_{1}}=0$. Then taking the derivative of $V_{2}$ along the trajectories of the system,

$$
\begin{aligned}
\dot{V}_{2} & =e_{s_{2}} \dot{e}_{s_{2}} \\
& \leq-\lambda_{s_{2}}\left|e_{s_{2}}\right|+\left|e_{s_{2}}\right|\left|-\frac{k_{s}}{m_{s}} e_{s_{1}}-\frac{b_{s}}{m_{s}} e_{s_{2}}-\frac{1}{m_{s}} F_{e}\right| \\
& =-\left|e_{s_{2}}\right|\left(\lambda_{s_{2}}-\left|-\frac{k_{s}}{m_{s}} e_{s_{1}}-\frac{b_{s}}{m_{s}} e_{s_{2}}-\frac{1}{m_{s}} F_{e}\right|\right)
\end{aligned}
$$

Then choosing,

$$
\lambda_{s_{2}}>\left|-\frac{k_{s}}{m_{s}} e_{s_{1}}-\frac{b_{s}}{m_{s}} e_{s_{2}}-\frac{1}{m_{s}} F_{e}\right|+\varepsilon_{2}
$$

ensures that $\dot{V}_{2} \leq-\left|e_{s_{2}}\right| \varepsilon_{2}<0$, for all $e_{s_{2}} \neq 0$. This choice of gain is possible due to Assumption 3.1 which ensures that the force $F_{e}$ is bounded. Finite time convergence to the surface $e_{s_{2}}=0$ is achieved by the Comparison Lemma. It can be verified that an upper bound on the time to reach the sliding surface $e_{s_{2}}=0$ is given as,

$$
T_{s_{2}} \triangleq \frac{\left|e_{s_{2}}\left(T_{s_{1}}\right)\right|}{\varepsilon_{2}}+T_{s_{1}}
$$

At this point, an estimate of the environmental force may be obtained. Solving for the equivalent output injection when $e_{s_{2}}=\dot{e}_{s_{2}}=0$ yields $\left(\lambda_{s_{2}} \operatorname{sgn}\left(e_{s_{2}}\right)\right)_{e q}=-\left(1 / m_{s}\right) F_{e}$. So the unknown environmental input is computed as,

$$
\hat{F}_{e}=-m_{s}\left(\lambda_{s_{2}} \operatorname{sgn}\left(e_{s_{2}}\right)\right)_{e q}
$$

This completes the portion of the proof showing finite time convergence of the state estimates to the true states and the availability of the environmental force estimate. The development for the master side observer is the same except that convergence times $T_{m_{1}}$ and $T_{m_{2}}$ are obtained 
for observer error states $e_{m_{1}}$ and $e_{m_{2}}$, respectively. The human force input on the master may be obtained as $\hat{F}_{h}=$ $m_{m}\left(\lambda_{m_{2}} \operatorname{sgn}\left(e_{m_{2}}\right)\right)_{e q}$.

To show stability of the master plant, substitute the master control law (18) into the master plant (8), (9). Simplifying, one arrives at,

$$
\begin{aligned}
\dot{x}_{m_{1}}= & x_{m_{2}} \\
\dot{x}_{m_{2}}= & -\frac{\bar{k}_{m}}{\bar{m}_{m}} \hat{x}_{m_{1}}-\frac{\bar{b}_{m}}{\bar{m}_{m}} \hat{x}_{m_{2}}+\frac{1}{\bar{m}_{m}} \hat{F}_{h} \\
& -\frac{1}{\bar{m}_{m}} \hat{F}_{e}^{d}-\frac{k_{m}}{m_{m}} e_{m_{1}}-\frac{b_{m}}{m_{m}} e_{m_{2}} \\
& +\frac{1}{m_{m}}\left(F_{h}-\hat{F}_{h}\right)
\end{aligned}
$$

The master dynamics will remain in this form until time $t=\max _{t}\left(T_{m_{2}}, T_{s_{2}}\right)$ when the observers converge. At this time the states of the master plant are finite and the observer errors go to zero. Then, the closed loop master dynamics become,

$$
\begin{aligned}
\dot{x}_{m_{1}}= & x_{m_{2}} \\
\dot{x}_{m_{2}}= & -\frac{\bar{k}_{m}}{\bar{m}_{m}} x_{m_{1}}-\frac{\bar{b}_{m}}{\bar{m}_{m}} x_{m_{2}}+\frac{1}{\bar{m}_{m}} F_{h} \\
& -\frac{1}{\bar{m}_{m}} F_{e}^{d}
\end{aligned}
$$

That is, after observer convergence the master manipulator has the desired impedance characteristic and is stable.

To show stability of the slave plant the goal is to show finite time convergence to the set $\{\hat{s} \leq \epsilon\}$. Consider the Lyapunov function candidate,

$$
V_{s}=\frac{1}{2} \hat{s}^{2}
$$

Taking the derivative of $V_{s}$ along the trajectories of the system,

$$
\begin{aligned}
& \dot{V}_{s}=\hat{s} \dot{\hat{s}} \\
& =\hat{s}\left[\left(\frac{\bar{k}_{s}}{\bar{m}_{s}}-\frac{k_{s}}{m_{s}}\right) \hat{x}_{s_{1}}+\left(\frac{\bar{b}_{s}}{\bar{m}_{s}}-\frac{b_{s}}{m_{s}}\right) \hat{x}_{s_{2}}\right. \\
& +\left(\frac{\bar{k}_{m}}{\bar{m}_{m}}-\frac{\bar{k}_{s}}{\bar{m}_{s}}\right) \hat{x}_{m_{1}}^{d}+\left(\frac{\bar{b}_{m}}{\bar{m}_{m}}-\frac{\bar{b}_{s}}{\bar{m}_{s}}\right) \hat{x}_{m_{2}}^{d} \\
& -\left(\frac{1}{\bar{m}_{m}}-\frac{1}{m_{m}}\right) \hat{F}_{h}^{d}+\frac{1}{\bar{m}_{m}} \hat{F}_{e}^{d d}-\frac{1}{\bar{m}_{s}} \hat{F}_{e} \\
& +\frac{1}{m_{s}} u_{s}+\lambda_{s_{2}} \operatorname{sgn}\left(\tilde{x}_{s_{2}}-\hat{x}_{s_{2}}\right) \\
& \left.-\lambda_{m_{2}} \operatorname{sgn}\left(\tilde{x}_{m_{2}}^{d}-\hat{x}_{m_{2}}^{d}\right)\right]
\end{aligned}
$$

Outside of the boundary layer, that is when $|\hat{s}|>\epsilon$, $\operatorname{sat}(\hat{s} / \epsilon)=\operatorname{sgn}(\hat{s})$. So when the sliding surface is outside of the boundary layer, the $\operatorname{sat}(\cdot)$ term in the control may be expressed as a $\operatorname{sgn}(\cdot)$ term. Substituting the slave control law (21) into (42) and simplifying, one arrives at,

$$
\begin{aligned}
\dot{V}_{s} \leq \quad & -|\hat{s}|\left(K_{g}-\mid \lambda_{s_{2}} \operatorname{sgn}\left(\tilde{x}_{s_{2}}-\hat{x}_{s_{2}}\right)\right. \\
& -\left(\lambda_{s_{2}} \operatorname{sgn}\left(\tilde{x}_{s_{2}}-\hat{x}_{s_{2}}\right)\right)_{e q} \\
& -\left(\lambda_{m_{2}} \operatorname{sgn}\left(\tilde{x}_{m_{2}}-\hat{x}_{m_{2}}\right)\right. \\
& \left.\left.+\left(\lambda_{m_{2}} \operatorname{sgn}\left(\tilde{x}_{m_{2}}-\hat{x}_{m_{2}}\right)\right)_{e q}\right) \mid\right)
\end{aligned}
$$

for $|\hat{s}|>\epsilon$. So choosing,

$$
\begin{aligned}
K_{g}>\quad \mid \lambda_{s_{2}} \operatorname{sgn}\left(\tilde{x}_{s_{2}}-\hat{x}_{s_{2}}\right)-\left(\lambda_{s_{2}} \operatorname{sgn}\left(\tilde{x}_{s_{2}}-\hat{x}_{s_{2}}\right)\right)_{e q} \\
\quad-\left(\lambda_{m_{2}} \operatorname{sgn}\left(\tilde{x}_{m_{2}}-\hat{x}_{m_{2}}\right)\right. \\
\left.\quad+\left(\lambda_{m_{2}} \operatorname{sgn}\left(\tilde{x}_{m_{2}}-\hat{x}_{m_{2}}\right)\right)_{e q}\right) \mid+\varepsilon_{g}
\end{aligned}
$$

where $\varepsilon_{g}>0$ ensures that $\dot{V}_{s} \leq-|\hat{s}| \varepsilon_{g}$ when $|\hat{s}|>\epsilon$. Then, $\dot{V}_{s}<0$, for all $|\hat{s}|>\epsilon$. This result ensures finite time convergence of the trajectories to the set $\{\hat{s} \leq \epsilon\}$ Once the system reaches the boundary layer it will remain in there for all time [12]. The use of the boundary layer results in some small deviation from the desired behaviour that would be achieved if the sliding surface $\hat{s}=0$ were reached. The magnitude of this deviation is directly related to the value chosen for $\epsilon$. The advantage, though, is that within the boundary layer a smooth control action is applied to the plant, which eliminates the effect of chattering found in ideal sliding mode control [12].

It can be shown using the Comparison Lemma that the time for the system trajectories to reach the set $\{\hat{s} \leq \epsilon\}$ is given by,

$$
T_{g} \triangleq \frac{\left|\hat{s}\left(t_{0}\right)\right|}{\varepsilon_{g}}+t_{0}-\frac{\epsilon}{\varepsilon_{g}}
$$

By time $t=\max _{t}\left(T_{s_{2}}, T_{m_{2}}, T_{g}\right)$ both sliding mode observers will have reached their respective sliding manifolds and the slave trajectories will have reached the set $\{\hat{s} \leq \epsilon\}$. At this point $\hat{s}=s$ and the characteristic slave impedance estimate will be equal to the desired characteristic slave impedance. That is, $\hat{I}=I$. The system then recovers the performance of a system where all states and forces are measured. Due to the linear nature of the plants, all states will be finite at time $t=\max _{t}\left(T_{s_{2}}, T_{m_{2}}, T_{g}\right)$ (i.e. the system does not have a finite escape time) and both the master and slave will be stabilized by their controllers at this point. Therefore, both plants achieve stable behaviour despite the presence of the time delay and with state and force estimates instead of measurements. The slave manipulator will track the delayed master position, while the master manipulator will experience a delayed version of the environmental force acting on the slave.

One can additionally show using a small gain argument that closed loop impedance parameters for the master and slave can always be chosen to ensure a stable closed loop system despite the presence of time delays. However, this proof is omitted here due to space constraints.

Having examined the proposed algorithm and shown its stability, Section IV will present a numerical example. 


\section{Simulation Results}

The performance of the algorithm proposed in this work is examined in this section through a simulation study. The master plant (8), (9) with master control (18) and observer (14), (15) is implemented. The slave dynamics (10), (11) with sliding mode controller (21) and observer (16), (17) are also implemented. Both master and slave have the same dynamic parameters of $m_{s}=m_{m}=5 \mathrm{~kg}, b_{s}=b_{m}=1.5$ $\mathrm{Ns} / \mathrm{m}$, and $k_{s}=k_{m}=1 \mathrm{~N} / \mathrm{m}$. The desired impedance for the master and slave is set the same in each of the controllers as well, with $\bar{m}_{s}=\bar{m}_{m}=5 \mathrm{~kg}, \bar{b}_{s}=\bar{b}_{m}=50 \mathrm{Ns} / \mathrm{m}$, and $\bar{k}_{s}=\bar{k}_{m}=125 \mathrm{~N} / \mathrm{m}$.

The sliding mode observer gains are chosen as $\lambda_{s_{1}}=$ $\lambda_{m_{1}}=10, \lambda_{s_{2}}=\lambda_{m_{2}}=20$. The sliding mode controller gain is chosen as $K_{g}=50$, which is large enough to ensure that a sliding mode occurs. In order to obtain the equivalent output injection signals in the sliding mode observers, two first order filters are used in each observer. The first filter in each observer has a bandwidth of $628 \mathrm{rad} / \mathrm{sec}$. The second filter in each observer has a bandwidth of $30 \mathrm{rad} / \mathrm{sec}$. The filter bandwidths must be selected such that the lower frequency equivalent signals are passed through while the higher frequency switching components are filtered out [13]. The human force applied to the master manipulator is a sine wave with amplitude of $20 \mathrm{~N}$, frequency of $2 / 3 \mathrm{rad} / \mathrm{sec}$, and a phase shift of $-\pi / 4$. The environment on the slave side is modeled as a spring at a position of $0.1 \mathrm{~m}$ with a stiffness of $K_{e}=150 \mathrm{~N} / \mathrm{m}$.

In order to show that the system functions in the presence of time delays, a time delay of 0.25 seconds is used in each direction, for a round trip time delay of 0.5 seconds. Both the master and slave manipulators are started with the initial conditions $x_{m}=x_{s}=[0,0]^{T}$. To show convergence of the observer error dynamics, both the master and slave sliding mode observers are given initial conditions of $\hat{x}_{m}=\hat{x}_{s}=$ $[0.1,0]^{T}$. The simulation is implemented in $\mathrm{C}$ using a fixed step fifth order Runge-Kutta solver to integrate the dynamics. The controller and plant simulations are run at a sample period of $T_{o}=1 \mathrm{msec}$. The sliding mode observers are run at a sample period of $T_{i}=10 \mu \mathrm{sec}$. The sliding mode observers are run at a higher rate in order to minimize the numerical error due to the discontinuous switching terms [14].

Figure 1 shows the performance of the system in terms of the master and slave positions. It is clear that when the slave is in free motion it accurately tracks the delayed master position. When the slave comes into contact with the environment, at $x_{s}=0.1 \mathrm{~m}$, the slave no longer tracks the master reference signals, as expected. Instead, the external force acting on the slave grows and is transmitted back to the master side. When the slave manipulator leaves contact with the environment it resumes tracking the delayed master position. The slave environmental force is shown in Figure 2. The force reflected back to the master is zero when the slave is in free motion, as desired. This is in contrast to position-position architectures such as that of Niemeyer and Slotine [4] where the tracking error is reflected back to the

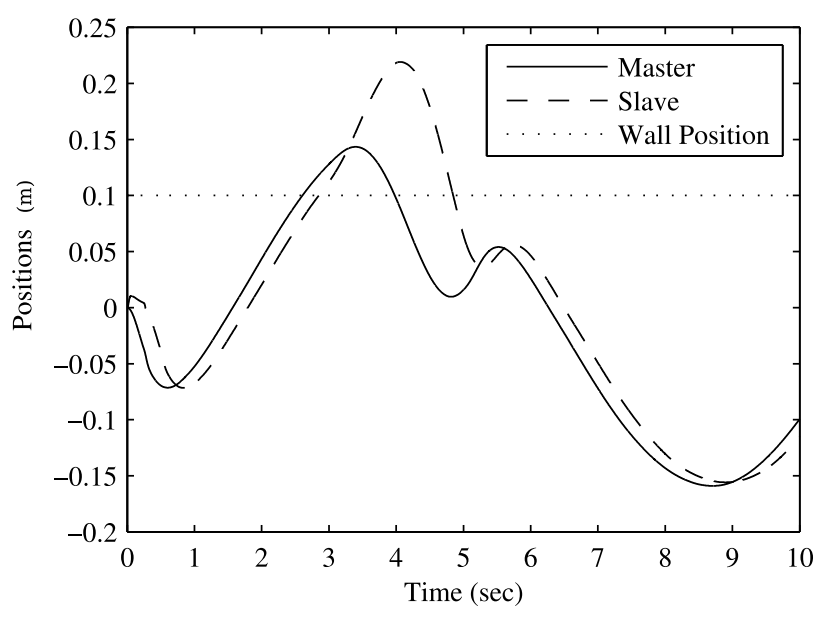

Fig. 1. Position of the master and slave manipulators.

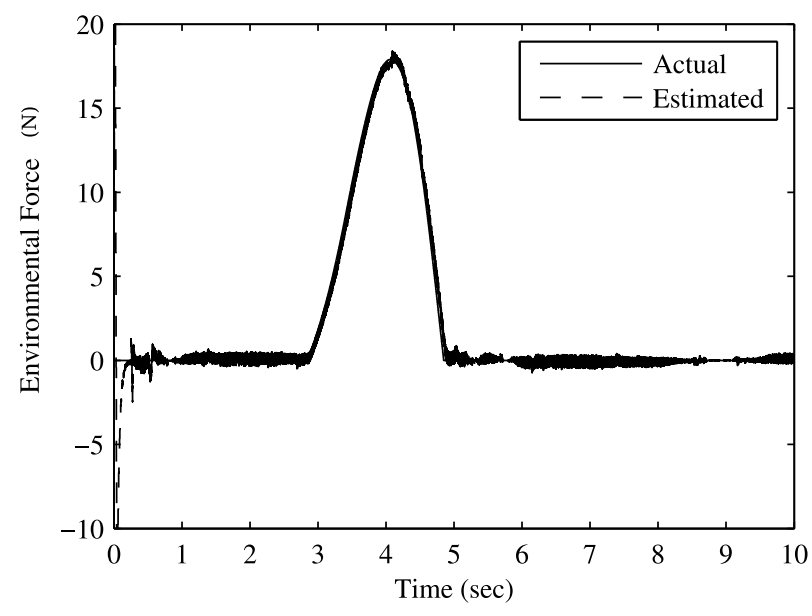

Fig. 2. Actual and estimated slave environment force.

master side, having the effect that even when the slave is in free motion it is possible that a force gets reflected back to the master side.

Examining Figure 2, once can see the effectiveness of the force estimation scheme. There is some initial peaking and error in the force estimate before convergence of the observer error dynamics to the sliding surfaces. The force applied by the human, along with its estimate, is shown in Figure 3. The same behaviour is observed in this plot. There is an initial peak in the estimate, but convergence to the actual force signal occurs in a short amount of time - less than 0.5 seconds. As seen in the plots, the accuracy of these force estimates justifies their use in the controllers instead of force measurements. Note that some high frequency components of low amplitude remain in the force estimates, but these components are effectively filtered out by the master and slave plants.

The simulation results presented in this section have shown that using unknown input observers to estimate the state of, and external force applied to, the robot manipulators is an effective alternative to actual force measurements. Through 


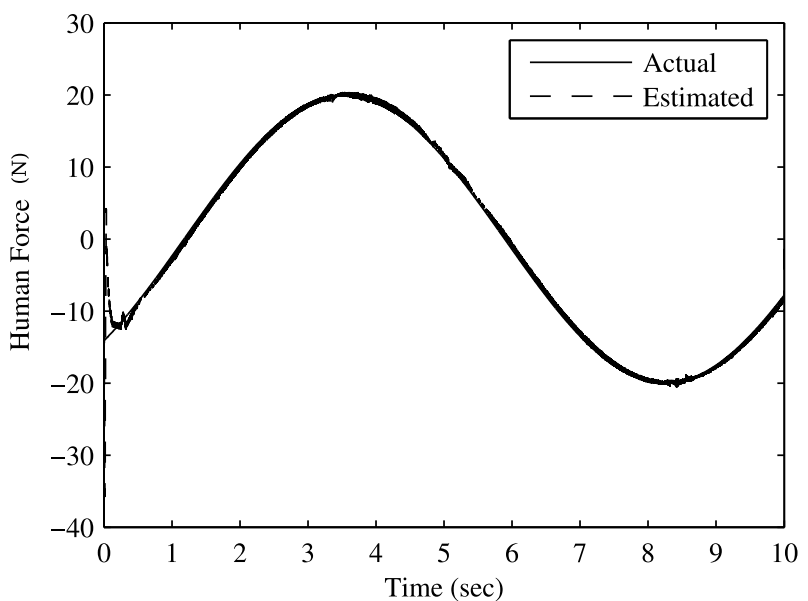

Fig. 3. Actual and estimated human applied force.

a numerical example, the system has been shown to be feasible.

\section{CONCLUSION}

This work has presented a new approach to control of a bilateral teleoperation system using force estimates instead of the actual force measurements. By modifying the control approach of Cho et al. [1] in such a way that only position measurements are required at the master and the slave sides and using unknown input sliding mode observers to estimate the forces, an algorithm with all of the desirable properties of both the position-position and position-force architectures is achieved. That is, one does not need to measure any of the forces on either manipulator, but the use of force estimates ensures that the increased performance of a position-force architecture is obtained. This new approach was shown to be stable and its feasibility was verified through simulation results. The results showed particularly that accurate estimates of the human and environment forces can be obtained and used for feedback.

This work will be further studied on the experimental side. Experimental validation of this technique is currently underway. As well, an examination of situations involving unmodeled friction in the plants and its effect on force estimation will be carried out to further ensure the practical implementability of this approach when portions of the dynamic model are not well known.

\section{REFERENCES}

[1] H. C. Cho, J. H. Park, K. Kim, and J.-O. Park, "Sliding-mode-based impedance controller for bilateral teleoperation under varying time delay," in Proceedings of the 2001 IEEE International Conference on Robotics \& Automation, May 2001.

[2] T. B. Sheridan, "Space teleoperation through time delay: Review and prognosis," IEEE Transactions on Robotics and Automation, vol. 9, pp. 592-606, Oct. 1993.

[3] D. A. Lawrence, "Stability and transparency in bilateral teleoperation," IEEE Transactions on Robotics and Automation, vol. 9, no. 5, pp. 624 637, Oct. 1993.

[4] G. Niemeyer and J.-J. E. Slotine, "Telemanipulation with time delays," The International Journal of Robotics Research, vol. 23, pp. 873-890, Sept. 2004.

[5] M. Tavakoli, A. Aziminejad, R. V. Patel, and M. Moallem, "Enhanced transparency in haptics-based master-slave systems," in Proceedings of the 2007 American Control Conference, July 2007.

[6] T. Floquet and J. P. Barbot, "A sliding mode approach of unknown input observers for linear systems," in Proceedings of the 43rd IEEE Conference on Decision and Control, Dec. 2004.

[7] J. H. Park and H. C. Cho, "Sliding-mode controller for bilateral teleoperation with varying time delay," in Proceedings of the 1999 IEEE/ASME Conference on Advanced Intelligent Mechatronics, Sept. 1999.

[8] M. Hou and P. C. Muller, "Design of observers for linear systems with unknown inputs," IEEE Transactions on Automatic Control, vol. 37, no. 6, pp. 871-875, June 1992.

[9] C. Edwards, E. F. Colet, and L. Fridman, Eds., Advances in Variable Structure and Sliding Mode Control. Heidelberg, Germany: SpringerVerlag, 2006.

[10] J. P. Barbot, T. Boukhobza, and M. Djemai, "Sliding mode observer for triangular input form," in Proceedings of the 35th Conference on Decision \& Control, Dec. 1996, pp. 1489-1490.

[11] S. Drakunov and V. Utkin, "Sliding mode observers. tutorial." in Proceedings of the 34th Conference on Decision \& Control, Dec. 1995, pp. 3376-3378.

[12] H. K. Khalil, Nonlinear Systems, 3rd ed. New Jersey: Prentice Hall Inc., 2002.

[13] I. Haskara, U. Ozguner, and V. Utkin, "On sliding mode observers via equivalent control approach,” International Journal of Control, vol. 71, no. 6, pp. 1051-1067, 1998.

[14] I. A. Shkolnikov, Y. B. Shtessel, and S. V. Plekhanov, "Multi-rate digital design for sliding-mode-observer-based feedback control," in Proceedings of the 2005 American Control Conference, June 2005. 